Article

\title{
An Energy Demodulation Based Fiber Optic Sensing System for Landslide Early-Warning
}

\author{
Xing Wang ${ }^{1}$, Bin Shi ${ }^{1, *}$, Guangqing Wei ${ }^{2}$ and Shenen Chen ${ }^{3}$ \\ 1 School of Earth Sciences and Engineering, Nanjing University, Nanjing 210046, China; 465802859@163.com \\ 2 Suzhou Nanzee Sensing Technology Co., Ltd., Suzhou 215123, China; wgq@nzsensing.com \\ 3 Department of Civil and Environmental Engineering, University of North Carolina at Charlotte, \\ Charlotte, NC 28223, USA; schen12@uncc.edu \\ * Correspondence: shibin@nju.edu.cn; Tel.: +86-25-8359-6220
}

Academic Editor: Christoph Peter Hauri

Received: 24 March 2017; Accepted: 9 June 2017; Published: 7 July 2017

\begin{abstract}
To help reduce the impact of geo-hazards, an innovative landslide early-warning technology based on an energy demodulation-based fiber optic sensing (FOS-LW for short) technology, is introduced in this paper. FOS-LW measures the energy change in a sensing fiber at the segment of micro-bending, which can be caused by landslide movements, and automatically raises an alarm as soon as the measured signal intensity in the fiber reaches a pre-set threshold. Based on the sensing of micro-bending losses in the fiber optics, a two-event sensing algorithm has been developed for the landslide early-warning. The feasibility of the FOS-LW technology is verified through laboratory simulation and field tests. The result shows that FOS-LW has some unique features-such as the graded alarm, real-time responses, remote monitoring, low cost and passive optical network—and can be applied in the early-warning of landslides.
\end{abstract}

Keywords: landslides; fiber optic sensing system; early-warning; energy demodulation; event sensor

\section{Introduction}

Disasters associated with severe landslides are common world-wide. Landslides contribute thousands of deaths per year and economic losses from property damage in the billions of dollars [1-3]. Although numerous attempts at monitoring the landslides $[4,5]$ have been undertaken since the early 1900s to evaluate the stability of the landslides. Conventional methods for landslide early-warning include inclinometer, displacement meter, strain gauges, water level meter, photogrammetry, and others [6]. These warning tools are point-monitoring or small-scale, sometimes, which cannot acquire the sufficient key signals for early-warning of a landslide [7].

It is difficult for current technologies to forecast the occurrence location, time, and intensity of displacement accurately. For the mitigation and prevention of landslide disasters, it is important to develop an effective early warning system for landslides. Slope stability is mainly affected by three basic factors, namely geo-structural, environmental, and human-induced (artificial) factors. Geo-structural factors that can trigger landslide events include the proximity to active faults, slide-prone geological formations, pre-existing fracture zones, degree of slope, degree of rock weathering, and the physical and mechanical parameters of the soil. Environmental factors may include the intensity and duration of a rainfall, and the movements of groundwater [8]. Man-made or artificial factors that add to these natural land-slide inducing elements may include activities such as blast vibrations, excavation, and transportation. No matter which element affects the stability of a slope, the outcome is the deformation of the slope. Thus, an effective early-warning of a landslide should focus on the deformation monitoring of the slope. 
In this paper, an innovative landslide early-warning technology is introduced. This technology is called energy demodulation based fiber optic sensing system for landslide warning (FOS-LW for short), and has built-in unique features such as graded alarm, real-time response, remote monitoring, and a passive optical networks [9-11]. It is able to monitor the early deformation of both soil and rock mass of slopes and thus serves as an early-warning technology for landslides.

\section{Principle behind FOS-LW}

The FOS-LW senses the deformation of the soil and rock mass through a change of light energy intensity in the fiber optic sensor cable. When the displacement resulted from deformation of the slope increases, the system identifies movements in the soil and rock from the change of signal energy intensity caused by localized micro bending in the cable. Figure 1 demonstrates the configuration of the FOS-LW system showing the signal generator sending out light into the sensing fiber. The light is modulated in the energy regulator, in which the light intensity is $I_{p}$. Through the displacement (event) sensors, a light signal is detected, which has an intensity of $I_{0}$. In the electro-optic modulator, the intensity change between the input light and output light is calculated as

$$
\Delta I=I_{p}-I_{o}
$$

where $\Delta I$ can be transmitted to the threshold judgment module. In the threshold judgment module, the data are compared with a pre-defined threshold, if the measured result is bigger than the threshold then the controller will send out a message to trigger the alarm; otherwise, the controller sends a message to the controller, which then commands the signal generator to send out the next round of light. The data can also be transferred to an analyzer for processing and analysis via Ethernet.

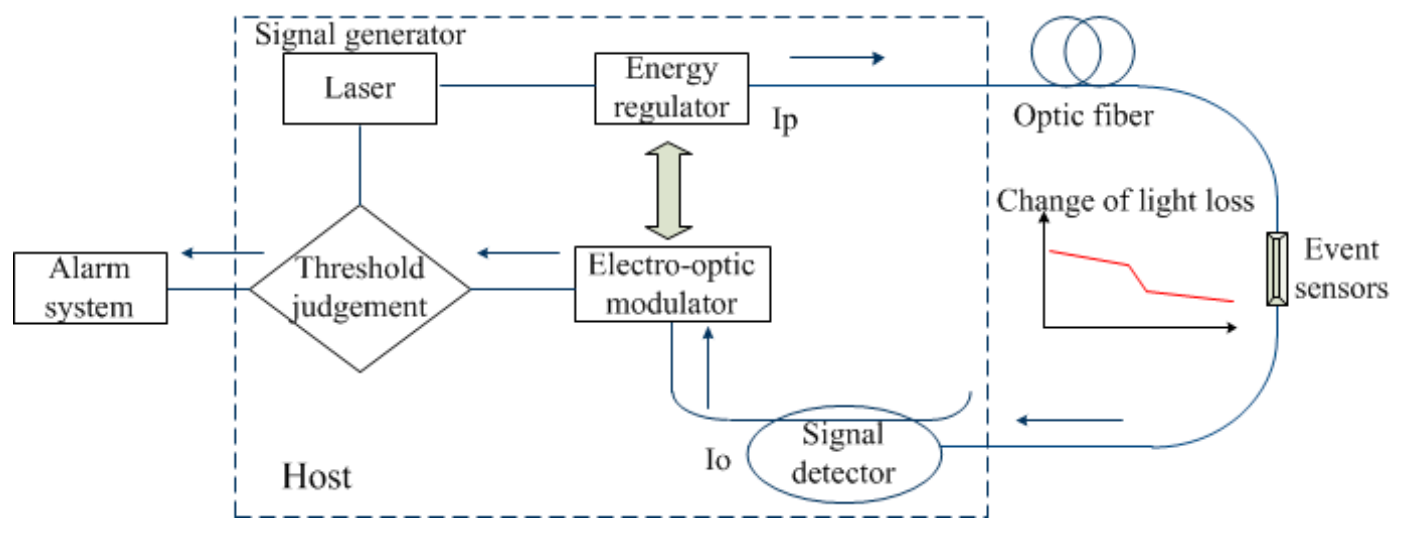

Figure 1. The schematic configuration of FOS-LW.

The key to the early warning system lies in establishing the relationship between the deformation of the landslide and the light intensity. Therefore, the event sensor is the key coupling of these two variables and a special linkage device is developed within the event sensor to enable the FOS-LW to detect the deformation of the landslide accurately.

\section{Event Sensors}

Different types of physical and mechanical parameters associated with landslides may require designs of different event sensors. Based on the principle of micro-bending, which is defined as the decrease in signal energy intensity in the fiber, two types of event sensors are developed: rack-type and spiral-type. 


\subsection{Rack-Type Sensor}

For a rack-type sensor, the micro-bending loss in an optical fiber will result from not satisfying the conditions for total internal reflection. When the degree of the bending exceeds the critical value in the fiber, the transmission mode is converted from conduction mode to radiation mode, causing light power consumption. Figure 2 demonstrates the schematic structure of the rack-type sensor. Figure $2 \mathrm{a}$ shows the initial state of the cable with the deformation apparatus. The deformation apparatus bites into the cable when it comes under an over-powering pressure, resulting in micro-bending of the cable. The greater the pressure is put on the deformation apparatus, the closer the two racks will bite into the cable, and the resulting light loss $A_{m}$ can be calculated as

$$
A_{m}=N<h^{2}>\frac{a^{4}}{b^{6} \Delta^{3}}\left(\frac{E}{E_{f}}\right)^{3 / 2}
$$

where $N$ : the number of micro bending; $h$ : the height of micro bending; $<>$ : statistical symbol; $E$ : the Young's modulus of the coating; $E_{f}$ : the Young's modulus of fiber; $a$ : the radius of the fiber; $b$ : the external radius of fiber; and $\Delta$ : the relative refractive index difference. The equation indicates that the micro bending loss has a strong relationship with the bending height for a given fiber [12].

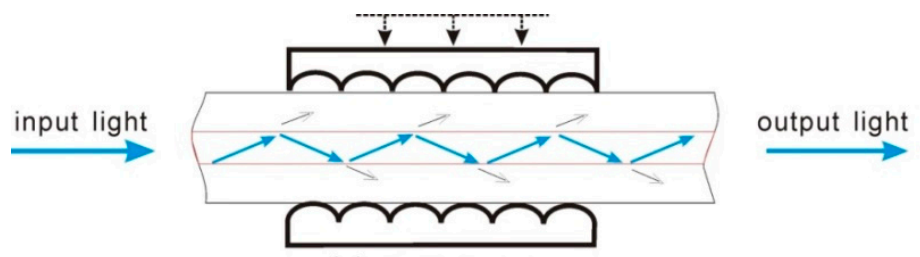

(a) initial state

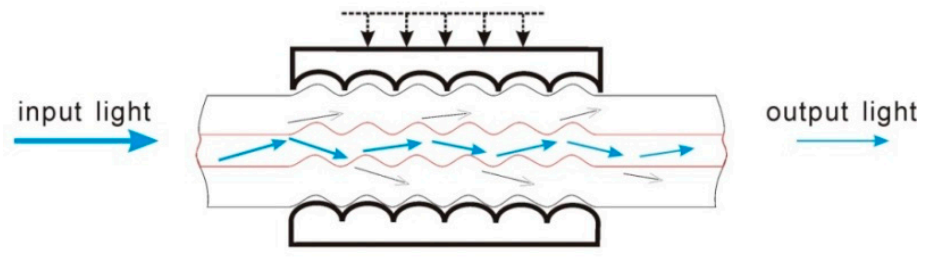

(b) operating state

Figure 2. Rack-type sensor (The blue arrow indicates the light transmission).

\subsection{Spiral-Type Sensor}

To improve the detection sensitivity, the micro bending fiber sensor is constructed as a spiral. Figure 3 demonstrates the structure of the spiral blazon sensor. The outer sheath can protect the fiber from being damaged. The movement of rock and soil is connected with the screw, which turns and winds the fiber cable tighter around the screw when movements are detected. The resulting light loss is associated with the radius of the screw and the loop number of the fiber cable around the screw. Hence, the bending loss of each coil winding is more consistent, allowing the relationship between the slope displacement and the output optical light intensity to remain linear. Thus, the deformation of the landslide and the displacement of the screw can be coordinated through the linkage system. 


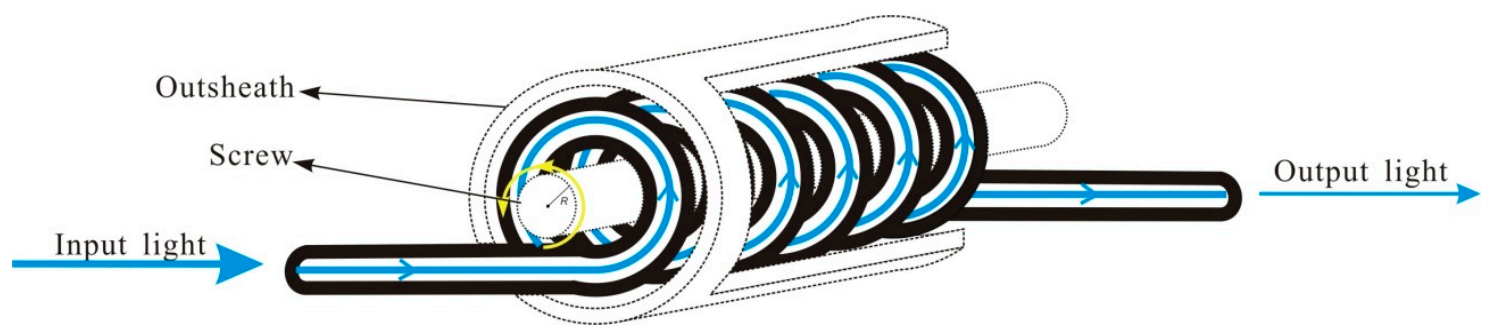

Figure 3. Schematic of spiral-type sensor (The blue arrow indicates the light transmission).

\section{The FOS-LW Components}

Figure 4 demonstrates a typical set-up of the optical fiber warning system, where the FOS-LW is integrated with a host device, an alarm system, an analysis module and power supply. The data is then transmitted to a remote control site. The integral system allows the realization of the FOS-LW system as an early warning system that can detect the landslide danger and send out a warning signal effectively.

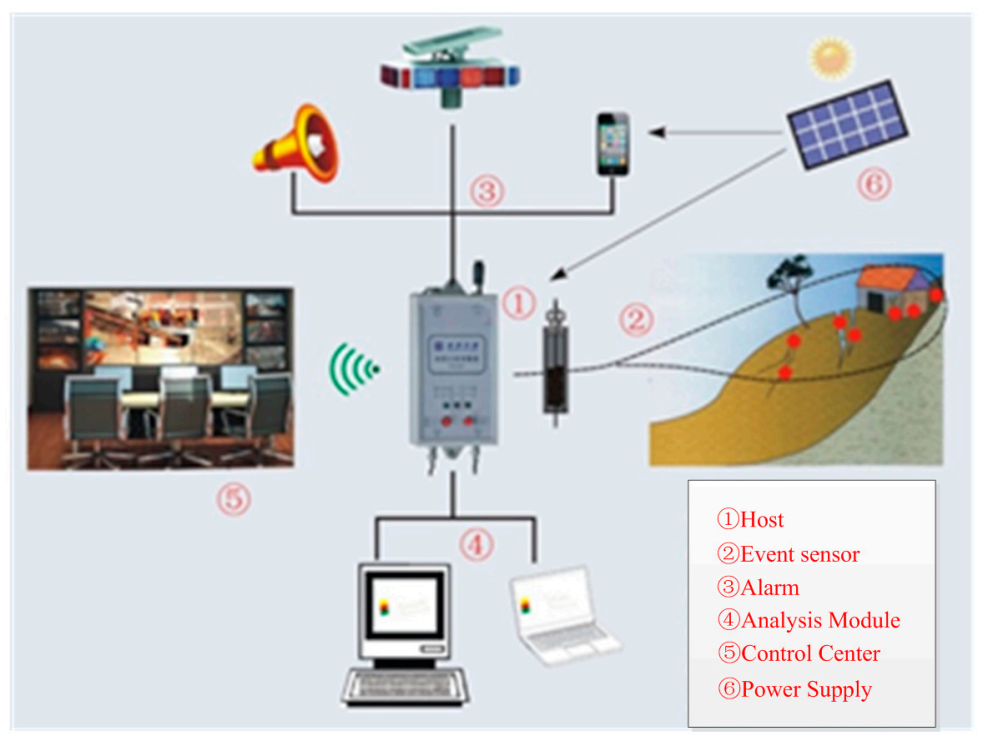

Figure 4. The physical set-up of FOS-LW.

\subsection{The Host}

Figure 5 shows the components and design of the host device. The host of the all-fiber landslide warning system is integrated with signal generator, the signal detector and the control module. The signal generator generates an optical signal, which can be a continuous signal, a pulse signal, or a pulse frequency modulated optical signal. These signals are connected with the (landslide) event sensors through the sensor cable. The signal detector is used for detecting the intensity of light through the event sensors. The main functions of the control module are to set the threshold value, to determine if the signal may be exceeding the threshold level, and to send out a decision command to the alarm module. 


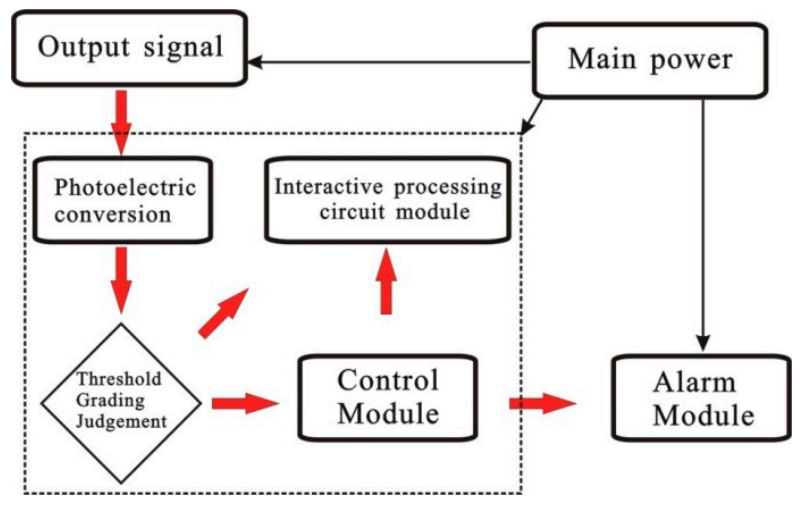

Figure 5. The signal transmission flow and the relationship between each integrated modules.

\subsection{The Alarm System}

A critical component of the alarm system is to set up an appropriate threshold level. FOS-LW used a graded threshold approach. Based on previous experiences, a typical landslide is expected to move slowly in the early stage and the movement will accelerate prior to full collapse. Figure 6 shows the different stages of a collapse with color coded to the corresponding alarm levels, which are described as follows:

- I-Creep Stage

The creep stage (green level) is a relatively safe stage, and during which, the sensors in the field should be monitored carefully with periodic inspections and analysis of the resulting data regularly.

- II-Acceleration Stage

At the acceleration stage (yellow level), the slope movement is picking up its pace, and there should be an increase in the frequency of data acquisition and review of the data, such that the underlying cause of slope movement can be determined through the data analysis. The most important aspect of this monitoring stage is to determine whether the threshold level should be maintained or lowered to ensure safety.

- $\quad$ III-Failure Stage

If the slope on the acceleration stage is out of control, and the slope deformation accelerates, the alarm system should raise the alarm level to red (warning level). At this stage, the engineers will be able to determine the proper courses of action either to minimize or eliminate possible economic and life losses associated with the slope failure.

It should be noted that the complete movement of a landslide can last for a few days or several years while the change in key factors may be very rapid. Because fiber optic sensors can be distributed and networked for different level conditions, the alarm system can be designed to send out specific alarm message in various ways: The alarm signal can be transmitted through sound and light warnings around the danger area. For distances further from the source of danger, the warning could be transmitted by text messaging via mobile devices. Remote scheduling and guiding work can be communicated to the system via wireless transmitting networks. Through this alarm system, one not only can warn of the danger, but also help to direct rescue efforts efficiently and remotely. 


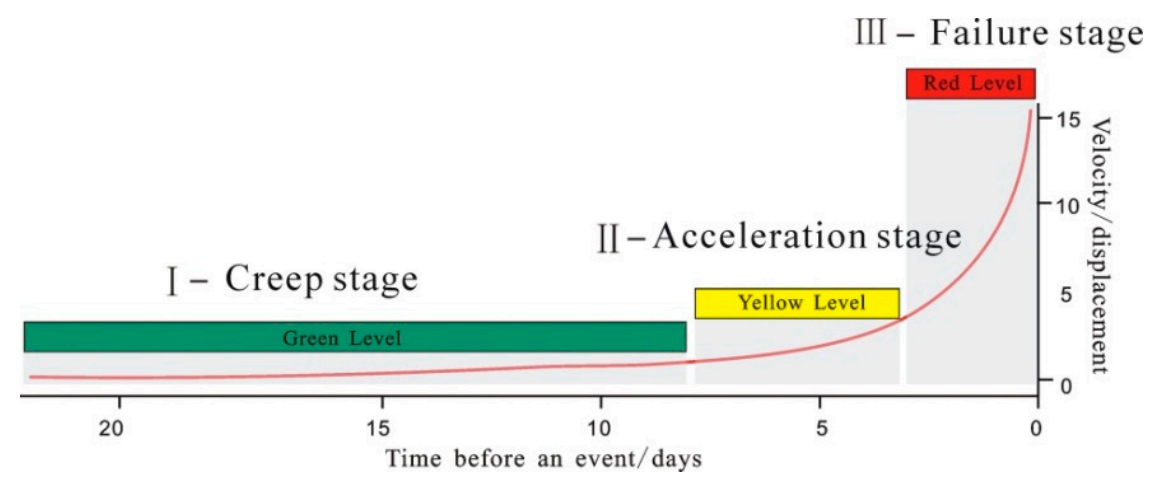

Figure 6. Three stages of the early-warning and the corresponding alarm level.

\subsection{The Analysis Module and Power Supply}

The data analysis module can assess the condition of each event (displacement) sensor according to the measured light loss, thus this module can provide the data support for monitoring the landslide. The power consumption of the whole framework and alarm is very small. Given that the locations of landslide zones are often remote, solar cells would be a good choice, and they can provide sufficient power for the whole system.

\section{Features}

The FOS-LW has features of real-time sensing and synchronization of event and warning, etc. In addition, the fiber consists of silica, which is anti-electromagnetic, resistant to corrosion, oxidation, and electrical interferences [13]. It is therefore suitable for the harsh environments in the field. The system also has other advantages.

\subsection{Grading the Demodulation Alarm}

Different geological conditions and external environments impact on landslides differently. A common reason for the high rate of false alarm in other systems is the lack of customizing to these conditions. The FOS-LW alarm threshold can be graded in multiple levels, with different alarms responding according to the specific grades. In this way, the accuracy of the early-warning system can be greatly improved. Meanwhile, the system does not measure specific values of the deformation; thus, significantly reducing the computing efforts behind the scene. As soon as the light intensity is lower than the threshold level, the system alarm is triggered.

\subsection{The Local Monitoring and Early-Warning Networks}

Landslides generally occur in some remote districts. Some landslides are very large, whereas others occur in the form of multiple landslides that can be clustered into groups. In certain areas, the FOS-LW can be integrated into a monitoring net through a single cable, forming a regional network. In this way, the efficiency and management of landslide monitoring and early-warning can be greatly improved.

\subsection{Low Cost of the Entire System}

The system does not need expensive modulation and demodulation devices and as the sensing medium, the optical fiber is also low cost. All in all, the cost of the entire system is just a fraction of the costs of conventional methods. Easy installation and precise warning makes the system more efficient and reliable than other methods. 


\section{Experimental Verification of FOS-LW}

The following experiments are for the calibration of spiral blazon sensor and the verification of the all-fiber warning system.

\subsection{Calibration of Spiral Blazon Sensor}

Before it is applied in the field, the spiral blazon sensor should be calibrated first. A $0.9 \mathrm{~mm}$ polyurethane sheath optical fiber cable is the best option for this lab experiment, and the cable is wound around the screw of the spiral blazon sensor as shown in Figure 3. The length of the cable wound on the screw is measured by metric ruler. For length of each circle to be $l=2 \pi R$, so the number of cylinder $x$ is determined as

$$
x=L / l=L / 2 \pi R
$$

where $L$ is the displacement of sensor; $R$ is the radius of the screw. The light loss is measured by an optical power meter. Figure 7 shows the relationship between cylinder number and light power. The $R^{2}$ of linear fitting equation is 0.99 . Thus, the linear relationship between light loss and cylinder number is established. The displacement data could be determined based on the measured light loss.

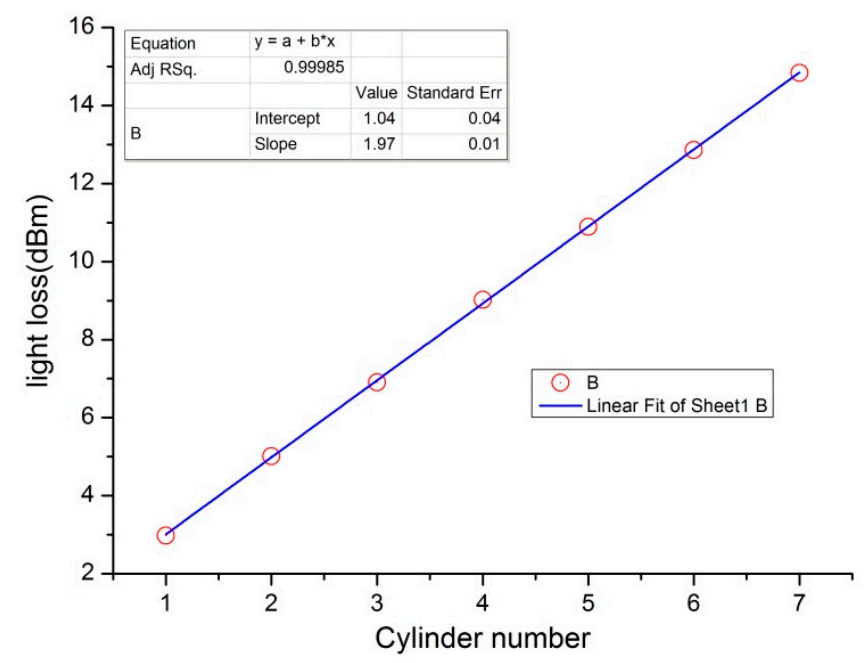

Figure 7. The linear relationship between light loss and cylinder number.

\subsection{Early-Warning Simulation Test}

The set-up of simulation experiment is demonstrated in Figure 8. The experimental setup involves mechanically induced displacement of the sensing system. The displacement meter is used to simulate the movement of a landslide. One end of the spiral blazon sensor is fixed to the displacement meter; the other end is controlled by the drive mechanism to simulate the deformation of landslides. The power supply provides electricity to the whole system, and the alarm function is accomplished by the alarm apparatus.

The preset measurement range of the displacement meter is $10.00 \mathrm{~cm}$ (corresponding to a $10.00 \mathrm{~cm}$ displacement of landslide). A three-level alarm system was set up. $0.00 \mathrm{~cm}$ (initial state) corresponds to the green level; $2.00 \mathrm{~cm}-8.00 \mathrm{~cm}$ corresponds to the yellow level; $8.00 \mathrm{~cm}-10.00 \mathrm{~cm}$ displacement corresponds the red level. The experimental procedure involved first making sure that the host was in working condition, the displacement meter was then slowly moved, representing the movement of the landslide. The dial indicator recorded the distance of the displacement meter movement. When the displacement meter reached about $2.00 \mathrm{~cm}$ degree, the alarm apparatus launched level one alarm response. As the displacement meter continued to about $8.00 \mathrm{~cm}$, the alarm apparatus launched level 
two alarm response. Given this very small error $(0.002 \mathrm{~cm})$ of the warning system, we can conclude that this system can be applied for in situ early-warning of landslides.
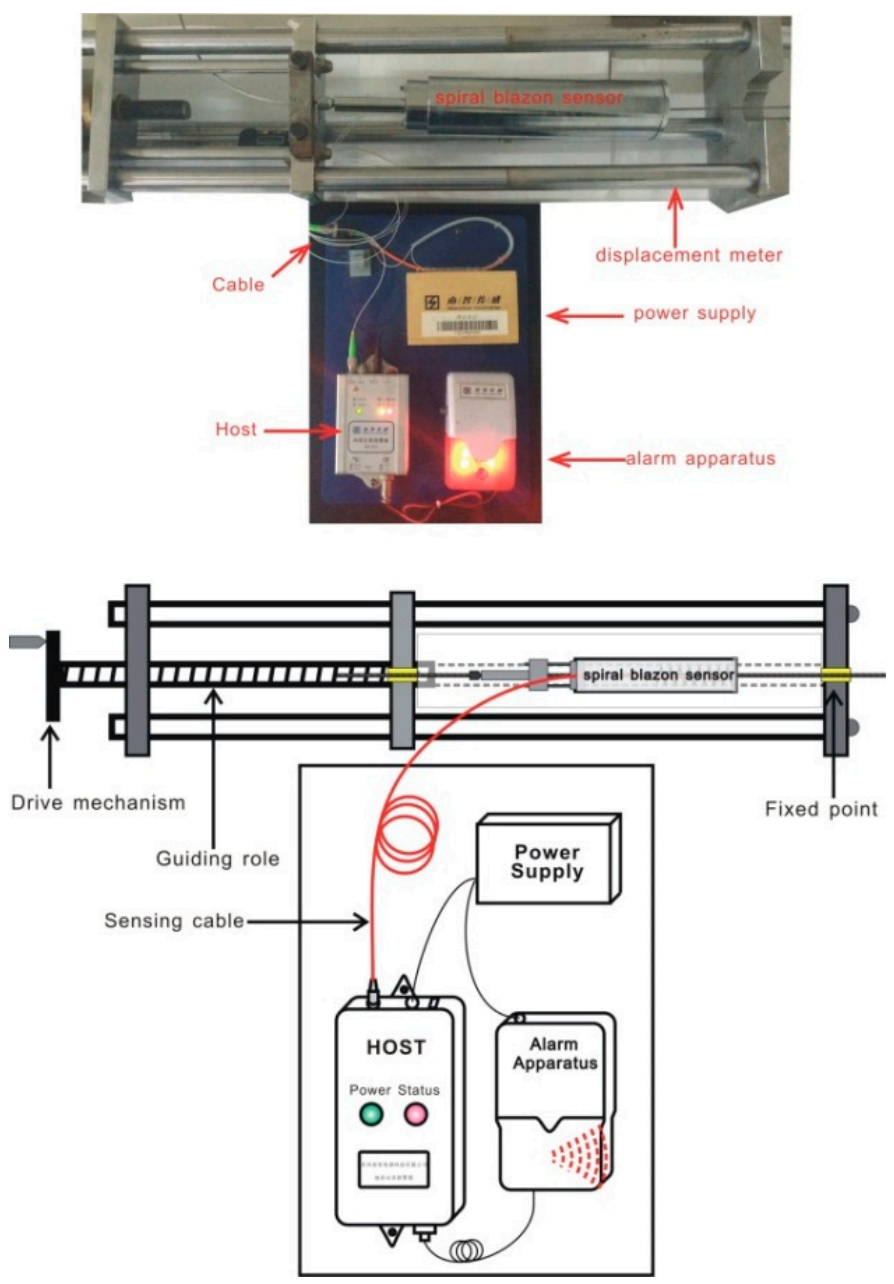

Figure 8. The warning system experimental set-up.

\section{The Threshold Determination}

The basic role of the threshold setting is to ensure that the system is neither too conservative, leading to numerous false alarms, nor fail to catch real events. The threshold setup should provide adequate warning time. The alarm thresholds determination of the FOS-LW was largely based on reviews of historical data and experiences with similar monitoring projects at other locations throughout the world. Based on extensive data analysis, the following three factors are concluded to determine the alarm thresholds, namely: horizontal displacement, vertical displacement, and the broadening of existing cracks [14]. For the FOW-LS system, two types of threshold are considered. Exceedance of these thresholds will result in an alarm announcement for the specific alarm level.

\subsection{Absolute Thresholds}

Absolute thresholds are mainly set based on the deformation of the landslides. As ongoing movement occurs, these values may vary according to the site conditions, so it may be necessary to reset the absolute threshold. Table 1 lists the absolute threshold values of a typical landslide. Different landslides may require different values according to site specific features. 
Table 1. The alarm class of absolute thresholds.

\begin{tabular}{cccc}
\hline Alarm Level & Horizontal Displacement & Vertical Displacement & Crack Width \\
\hline Green level & $<5.00 \mathrm{~mm}$ & $<3.00 \mathrm{~mm}$ & $<3.00 \mathrm{~mm}$ \\
Yellow level & $5.00 \mathrm{~mm} \sim 10.00 \mathrm{~mm}$ & $3.00 \mathrm{~mm} \sim 10.00 \mathrm{~mm}$ & $3.00 \mathrm{~mm} \sim 7.00 \mathrm{~mm}$ \\
Red level & $>10.00 \mathrm{~mm}$ & $>10.00 \mathrm{~mm}$ & $>7.00 \mathrm{~mm}$ \\
\hline
\end{tabular}

\subsection{Velocity-Based Thresholds}

Velocity-based thresholds consider the rate of slope movement or rate of change in the rock mass. These values are mainly expressed in $\mathrm{mm} /$ day or $\mathrm{mm} /$ week. Because of the complicated geological conditions and complex mechanisms involved in a landslide, velocity-based thresholds are often determined based on the experiences of a rock slope engineering specialist [15].

\section{Case Study-FOS-LW for Gaoqin Landslides}

To verify the feasibility of the FOS-LW, the system was installed to monitor the GaoQin landslides, located in the Huangshan Mountains, southern China. The slope of the ladder-shaped GaoQin landslides is about 18 to 25 degrees. According to preliminary reconnaissance, the landslide is covered with residual loose soil, and the underlying bedrock is badly weathered. As a result, rainwater can easily penetrate into the slope body, causing instability of slope. The landslide is at creep deformation stage at the time of this study. Around the slope body, a lot of cracks can be identified around the boundary of the landslide. Figure 9 shows the installation of the event sensors. The event sensors are installed in the slot with the fixture to guarantee the simultaneous deformation between the sensor and slope body.
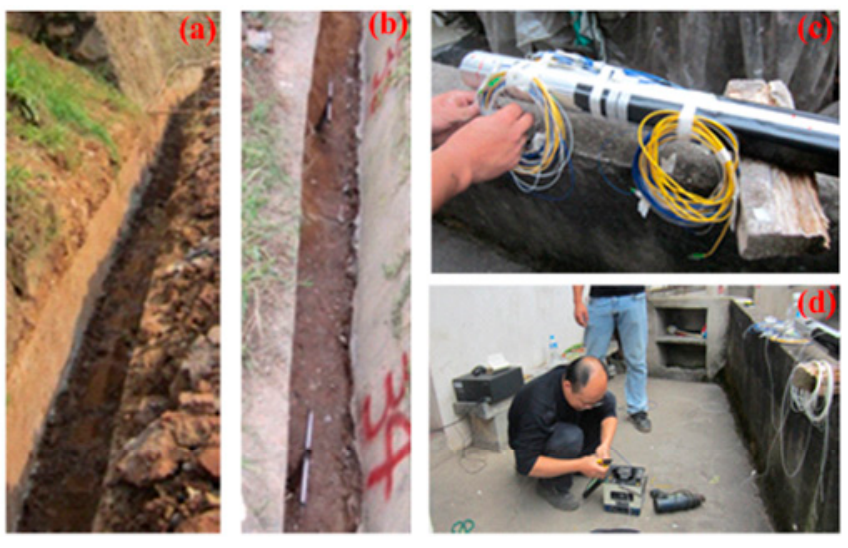

Figure 9. Site pictures: (a) slotting; (b) installation of fixture; (c) sensors with patch cord; (d) splicing of the fiber.

Figure 10 is the sensor layout of the FOS-LW system. Twelve event sensors were placed along the main sliding direction, which are used for the monitoring of horizontal and vertical displacements respectively. For comparison, an inclinometer was installed in a borehole extending to the bed rock beneath the slip surface in the central part of the landslide body to monitor the deformation of the landslide. After nearly a year of monitoring, this system is at the green level. According to the data acquired through the inclinometer $(0.5 \mathrm{~m}$ per point), as shown in Figure 11, the sliding displacement is less than $1 \mathrm{~mm}$, which is consistent with the warning status of the all fiber warning system. Figure 11 shows the horizontal displacements monitored throughout the study period. The maximum displacement of the whole slope is very small; which corresponds to the creep stage in Figure 6. 


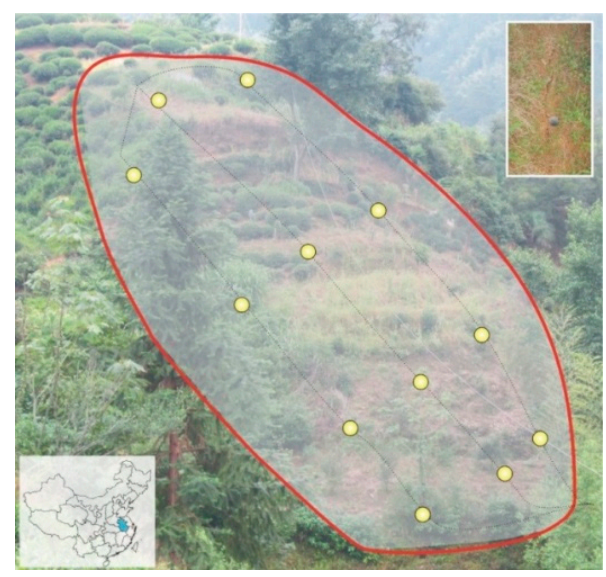

Figure 10. The sensor layout of Gaoqin landslide.

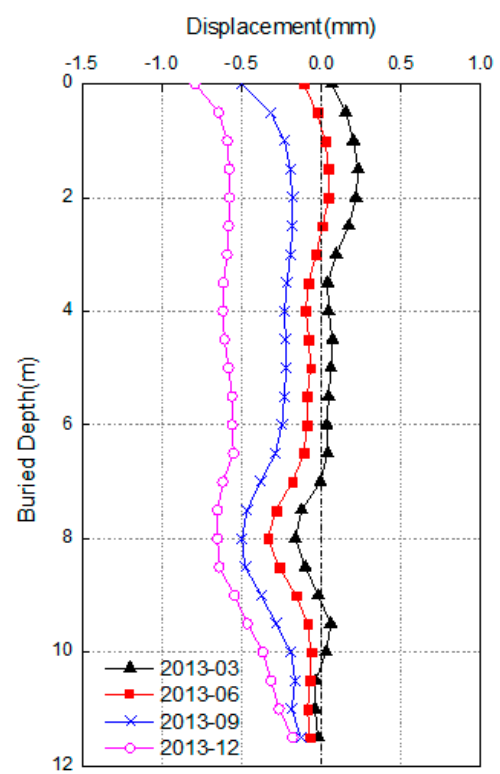

Figure 11. The horizontal displacement of landslide body.

\section{Conclusions}

Based on the energy demodulation principle in fiber optic sensing, an all fiber warning system is proposed. The FOW-LS system consists of the event (displacement) sensors, a host system, an alarm module, an analysis module, and power supply. The proposed system allows graded threshold levels for landslide warning and provides two novel event sensors: rack-type sensor and spiral blazon sensor. A laboratory experiment indicates that the spiral blazon sensor measured light intensity with a linear relationship between deformation and micro bending loss; thus, the sensor is stable for site monitoring.

Three conditions are considered in the threshold setting, namely, horizontal displacement, vertical displacement, and crack length. The alarm threshold considered two categories of monitoring strategy: absolute threshold and velocity-based threshold. In this paper, the absolute threshold of a typical landslide is determined.

The all fiber warning system has been applied to the Gaoqin landslide successfully. Based on the monitoring results of an inclinometer, the system correctly predicted the slope movements and shows that the proposed FOW-LS system can be used as a universal technology for the monitoring and early-warning of landslides with its unique advantages. 
Acknowledgments: This research is financially supported by the National Key Scientific Instrument and Equipment Development Projects, China (Grant No. 41230636).

Author Contributions: Bin Shi conceived and designed the study. Guangqing Wei assisted with the layout of the sensors in site and Shenen Chen's language editing work. Xing Wang wrote the paper. All authors read and approved the manuscript.

Conflicts of Interest: The authors declare no conflict of interest.

\section{References}

1. Hong, Y.; Adler, R.F.; Negri, A.; Huffman, G.J. Flood and landslide applications of near real-time satellite rainfall products. Nat. Hazards 2007, 43, 285-294. [CrossRef]

2. Yin, Y.; Wang, F.; Sun, P. Landslide hazards triggered by the 2008 Wenchuan earthquake, Sichuan, China. Landslides 2009, 6, 139-152. [CrossRef]

3. Devoli, G.; Strauch, W.; Chávez, G.; Høeg, K. A landslide database for Nicaragua: A tool for landslide-hazard management. Landslides 2007, 4, 163-176. [CrossRef]

4. Anderson, E.G.; Stoliker, P.C. Remote EDM Monitoring of Fractures on Turtle Mountain; Alberta Environment: Edmonton, AB, Canada, 1983.

5. Kostak, B.; Cruden, D.M. The moire crack gauges on the crown of the Frank Slide. Can. Geotech. J. 2011, 27, 835-840. [CrossRef]

6. Fraser, C. Photogrammetric monitoring of Turtle Mountain: A feasibility study. Free-network adjustment and sensitivity analysis are employed in the design of a deformation monitoring scheme. Photogramm. Eng. Remote Sens. 1983, 49, 1551-1559.

7. Kumar, A.; Sanoujam, M. Landslide studies along the national highway (NH 39) in Manipur. Nat. Hazards 2007, 40, 603. [CrossRef]

8. Guzzetti, F.; Peruccacci, S.; Rossi, M.; Stark, C.P. The rainfall intensity-duration control of shallow landslides and debris flows: An update. Landslides 2008, 5, 3-17. [CrossRef]

9. Shi, B.; Ding, Y.; Hongzhong, X.U.; Zhang, D. An Application of Distributed Optic Fiber Strain Measurement to Early-warning of Landslide. J. Eng. Geol. 2004, 12, 515-518.

10. Gao, L.; Shi, B.; Zhu, Y.; Wang, K.; Sun, Y.; Tang, C. A distributed soil temperature measurement system with high spatial resolution based on BOTDR. Opt. Appl. 2011, 41, 607-616.

11. Sun, A.; Semenova, Y.; Farrell, G.; Chen, B.; Li, G.; Lin, Z. BOTDR integrated with FBG sensor array for distributed strain measurement. Electron. Lett. 2010, 46, 66-68. [CrossRef]

12. Liu, Y.; Liu, J.P.; Zhu, Z.; Liu, Y. Experimental Research on Micro-bend fiber optica Pressure Sensor. Instrum. Tech. Sens. 2008, 1, 4-5.

13. Jiang, C.G.; Liu, F.J.; Dong, X.M.; Peng, J.G. Research and Manufacture on Integrated Monitoring System of Landslide; China Occupational Safety \& Health Management System Certification: Wuxi, Jiangsu, China, 2005.

14. Qiao, J. Probability Anlysis Based Method For Rainfall-induced Landslide Warning. J. Eng. Geol. 2009, 17, 343-348.

15. Crosta, G.B.; Agliardi, F. Failure forecast for large rock slides by surface displacement measure. Can. Geotech. J. 2003, 40, 176-191. [CrossRef]

(C) 2017 by the authors. Licensee MDPI, Basel, Switzerland. This article is an open access article distributed under the terms and conditions of the Creative Commons Attribution (CC BY) license (http:/ / creativecommons.org/licenses/by/4.0/). 